\title{
Antimicrobial resistance in Canada: An update on activities of the Canadian Committee on Antibiotic Resistance
}

John Conly MD FRCPC for the Canadian Committee on Antibiotic Resistance

$\mathrm{T}^{\mathrm{s}}$ his article is the first in a planned series of semi-annual updates, intended to provide readers with up-to-date information on antibiotic resistance in Canada and on the many current activities designed to address the problem. This information is provided by the Canadian Committee on Antibiotic Resistance (CCAR), a national organization that has a mandate to coordinate activities designed to address the growing problem of antibiotic resistance in Canada.

Antibiotic resistance has become an issue of increasing importance in recent years as more patients become infected with resistant pathogens, and as the public, the media and health care professionals become aware of the significant economic and social consequences of these diseases. The World Health Organization (1), the United States Centers for Disease Control and Prevention (2) and several other key organizations have released reports urging immediate action and providing recommendations for combatting this serious public health threat.

Today, clinically important pathogens such as methicillinresistant Staphylococcus aureus (MRSA), vancomycin-resistant enterococci (VRE), penicillin-resistant pneumococci and a host of other pathogens are placing an increasing economic, operational and social burden on health care facilities and communities around the world. To date, Canada has an enviable record of relatively low levels of resistance, although some regions of the country are experiencing rapidly rising rates of resistance.

\section{CCAR}

In 1997, Health Canada convened a consensus conference on antibiotic resistance to develop a coordinated national plan to address this problem. The result of the conference was the creation of an Integrated Action Plan for Canadians on Controlling Antimicrobial Resistance (3). In November 1999, Health Canada committed to three years of funding to support the activities of CCAR, which is charged with facilitating implementation of the Action Plan.

CCAR's efforts have been focused in three main areas: resistance surveillance, optimal antibiotic use, and infection prevention and control. Membership is open to not-for-profit organizations that, by the nature of their work, directly affect or influence the outcome of antibiotic resistance. These include professional societies, industrial associations, and government departments and agencies. As well, associate membership is available to individuals who are actively engaged in issues related to antibiotic resistance. CCAR represents over 20 health, medical, industry and government organizations. A full listing of member organizations and individuals is available at $<\mathrm{http}$ ://www.ccar-ccra.org/aboute.htm >. In addition to funding from Health Canada, CCAR is supported by educational grants from several of Canada's major health care companies.

CCAR's primary activities to date have concentrated on communications and outreach. These activities have includ- 
ed the development and distribution of information kits for physicians and veterinarians, provision of seminars to key target audiences and publication of an inventory of active antibiotic resistance programs in Canada. As well, CCAR hosted a workshop on resistance surveillance and provided a series of recommendations to Health Canada for developing a strong national surveillance program. Highlights include recommendations:

- to develop both active and passive surveillance activities;

- to integrate existing surveillance components with central processing and expanded cooperation;

- to expand existing systems to include sentinel laboratories, agrifood and veterinary data, and Aboriginal health information;

- to establish a steering committee to set priorities, select data sets of interest, provide guidance and management, and to establish a secretariat to review data and provide analysis;

- to establish mechanisms to standardize data provision, output and use criteria; and

- to include communications professionals in outreach activities.

CCAR has also created Canada's most comprehensive Web site on the issue (4). The Web site, which is continually updated, provides information on national and international activities, data on antibiotic utilization rates in Canada through an agreement with IMS Health Canada, extensive research references and links to many other organizations related to antibiotic resistance. The site also offers current trend reports of resistance patterns in Canada from various national and regional surveillance systems, either linked or posted on the site, providing a composite portrait of antibiotic resistance in Canada.

In January, CCAR announced an encouraging decline in the use of antibiotics in this country. In a widely distributed news release, CCAR reported that between 1995 and November 2000, Canadians saw a decrease of $14 \%$ in the overall number of antibiotic prescriptions dispensed, adjusted by population. Prescriptions of penicillins, tetracyclines and sulphonamides, as well as cephalosporins, all declined significantly when adjusted for changes in population over the past five years. Extended spectrum macrolides and quinolones, however, are being increasingly prescribed in Canada, indicating the need for continued efforts to promote appropriate prescribing. Inappropriate prescribing of antibiotics is recognized as a major factor in the emergence and spread of antibiotic resistance.

\section{FOCUS ON PREVENTION}

While CCAR has initially focused on surveillance and optimal antibiotic use, activities over the coming year will increasingly address the issues of the prevention of infection and control of transmission of antibiotic-resistant organisms. Workshops are planned for annual conferences in both the human health and agrifood sectors. Other plans include a report on the economic and social burden of illness resulting from antibiotic resistance, an information kit for pharmacists and a series of articles in key health care journals.

CCAR continues to warn that without coordinated, prompt national action, efforts to prevent the spread of resistant organisms will be ineffective, and the cost to the Canadian public will continue to grow.

"Pay now or pay later. It's that old adage that an ounce of prevention is worth a pound of cure," said CCAR chairman John Conly, commenting on the May outbreak of a new strain of multidrug-resistant Escherichia coli in the Durham region of Ontario, east of Toronto (5). "There's no systematic, governmental response" to antibiotic resistance, Dr Conly told The Globe and Mail (5). "It's something that's crying for attention from our federal and provincial governments." (5)

Health Canada estimates that it costs Canada's large teaching hospitals alone more than $\$ 35$ million a year to deal with multidrug-resistant organisms.

\section{ACTIVITIES OF CCAR ORGANIZATIONS}

The following are highlights of recent activities from selected CCAR-affiliated organizations.

Canadian Hospital Epidemiology Committee: The Canadian Hospital Epidemiology Committee (CHEC), a subcommittee of the Canadian Infectious Disease Society, in a collaborative effort with Health Canada, is providing rates and trends of nosocomial infections in sentinel hospitals within the Canadian Nosocomial Infection Surveillance Program (CNISP). Currently, there are 38 CHEC sites, representing nine provinces and 15 Canadian medical schools.

CNISP has carried out a number of projects examining the epidemiology of antimicrobial-resistant bacteria in participating CHEC hospitals. CHEC hospitals have reported the incidence of MRSA since 1995. A CHEC report summarizing the first five years of MRSA surveillance appears in a recent issue of the Canadian Medical Association Journal (6). CNISP has carried out several surveys for VRE in CHEC hospitals, and the results of the Canadian Passive Reporting Network for VRE will be published later this year. (An incidence survey for the occurrence of VRE in CHEC hospitals, comparable with the MRSA project, was implemented in 1998.)

Based on CHEC surveillance data, the epidemiology of antimicrobial-resistant bacteria in Canadian hospitals is quite different from that in other countries, especially the United States. MRSA was introduced into Canadian hospitals very late but is currently spreading rapidly. VRE was introduced into Canada in the mid-1990s, but unlike American hospitals where it has spread very rapidly, VRE has remained stable at low frequency through most of the country. 
Canadian hospitals have, to date, experienced minimal problems with ESBL-producing organisms, although these strains have reached an extremely high frequency in some American and western European hospitals.

Ontario Drug Programs Branch, Ministry of Health and Long Term Care: The Ontario Drug Programs Branch, Ministry of Health and Long Term Care has undertaken a variety of activities related to antibiotic resistance. The Ministry's expert drug advisory committee, the Drug Quality and Therapeutics Committee (DQTC), completed a review of all antibiotics listed in the Ontario Drug Benefit Formulary/ Comparative Drug Index (Formulary) to ensure that all antibiotics listed in the Formulary were being used appropriately in accordance with current clinical evidence. The Government of Ontario approved the changes to the formulary in January 2001, with an effective date of March 7, 2001. These documents are available on the Web at $<$ http://www.gov.on.ca/health/index.html>or by phone at 1-800-268-5559.

The most significant listing changes affected the quinolone antibiotics. The subcommittee was particularly concerned about increasing rates of resistance to this class of antibiotics and the spread of cross-resistance from older to newer quinolones. Thus, the listings for ciprofloxacin, levofloxacin and ofloxacin have been revised to ensure the most appropriate use of these agents and to limit the spread of resistance to this valuable class of agents. Activities to support this effort include a comprehensive public communication effort, and an information kit for physicians, pharmacists and nurse practitioners.

A drug utilization review will be conducted to monitor the changes to the Formulary listing within the coming year. Copies of the documents are available on the Web at $<$ http://www.gov.on.ca/health/index.html> or by phone at 1-800-268-5559.

Canadian Animal Health Institute: The Canadian Animal Health Institute (CAHI) is the trade association representing companies that develop and manufacture animal health products in Canada, including veterinary pharmaceuticals, biologicals, feed additives and animal pesticides. In 2000, CAHI produced a principles document, "Animal Health Use of Antimicrobials" (7), which details CAHI support of antimicrobials as beneficial health management tools for both companion and food animals. CAHI serves on the Advisory Committee on Non-Human Uses of Antimicrobials and Their Impacts on Resistance and Human Health. The committee was formed in 1999, and its members include delegates from the federal health ministry, academia, animal production sec- tors and their suppliers. This group is gathering relevant information pertaining to surveillance needs, veterinary drug approval program requirements, importation and use of nonapproved medications, and identification of gaps in knowledge. This information will then be used by the committee to formulate meaningful recommendations related to nonhuman use of antimicrobials.

Canadian Veterinary Medical Association: The Canadian Veterinary Medical Association (CVMA) has been very active in addressing the issue of responsible use of antibiotics in food animal production. To that end, the CVMA developed Guidelines for the Prudent Use of Antimicrobial Drugs in Animals (8). Also, in collaboration with CCAR, the CVMA distributed an information kit to all Canadian veterinarians to assist them in dealing with this issue. The CVMA is now looking at developing species-specific guidelines on the prudent use of antimicrobial drugs.

For more information about CCAR, please contact Rick Walter, Executive Director, Canadian Committee on Antibiotic Resistance, 3806 West 33 Avenue, Vancouver, British Columbia V6N 2H6. Telephone 613-260-3233, fax 613-260-3235, Web site http://www.ccar-ccra.org, e-mail cccar@home.com

ACKNOWLEDGEMENTS: Thank you to Rick Walter, Dodie Katzenstein and the executive of the Canadian Committee on Antibiotic Resistance for their contributions to this article.

\section{REFERENCES}

1. World Health Organization. World Health Organization Report on Infectious Diseases 2000: OvercomingAntimicrobial Resistance. <http://www.who.int/infectious-diseasereport/2000/> (Version current at July 31, 2001)

2. Centers for Disease Control and Prevention. A Public Health Action Plan to Combat Antimicrobial Resistance. <http://www.cdc.gov/drugresistance/actionplan/index.htm> (Version current at June 5, 2001)

3. Health Canada. An Integrated Action Plan for Canadians on Controlling Antimicrobial Resistance. <http://www.hc-sc.gc.ca/ hpb/lcdc/publicat/ccdr/97vol23/vol23s7/index.html> (Version current at August 3, 2001)

4. Canadian Committee on Antimicrobial Resistance $<$ http://www.ccra-ccar.org > (Version current at July 19, 2001)

5. Nelson M. Action needed on superbugs. The Globe and Mail May 30, 2001:A19.

6. Simor AE, Ofner-Agostini M, Bryce E, et al. The evolution of methicillin-resistant Staphylococcus aureus in Canadian hospitals: 5 years of national surveillance. CMAJ 2001;165:21-6.

7. Canadian Animal Health Institute. Animal Health Use of Antimicrobials. <http://www.cahi-icsa.ca> (Version current at June 8, 2001)

8. Guidelines for the Prudent Use of Antimicrobial Drugs in Animals. Ottawa: Canadian Veterinary Medical Association, 2000. 


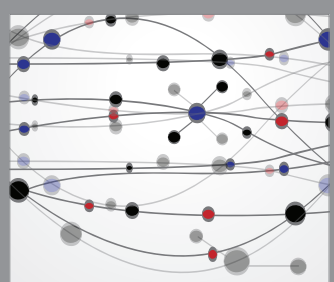

The Scientific World Journal
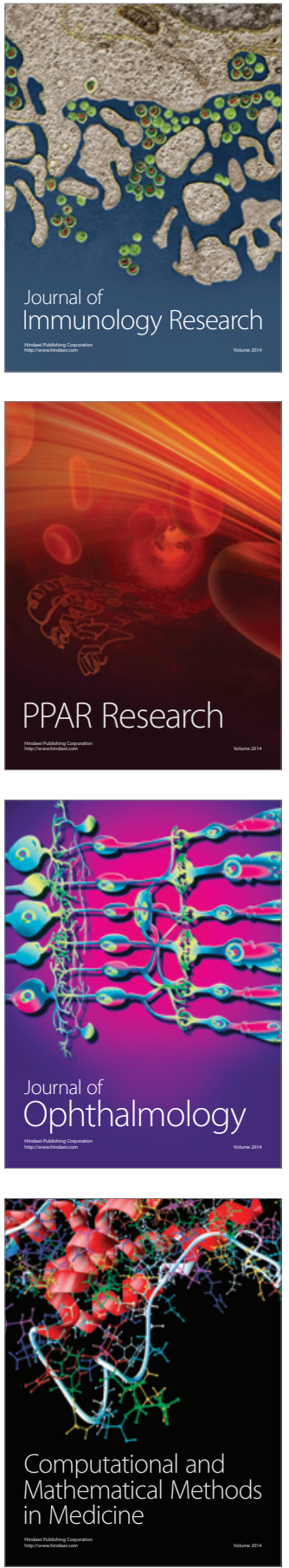

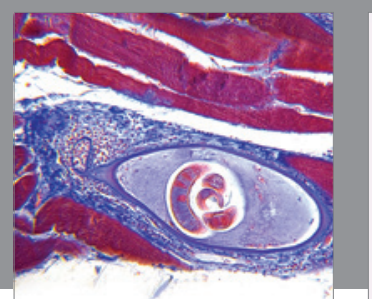

Gastroenterology Research and Practice

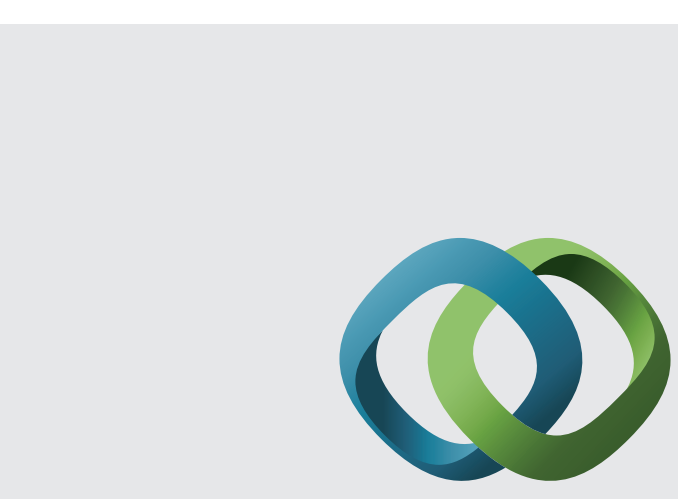

\section{Hindawi}

Submit your manuscripts at

http://www.hindawi.com
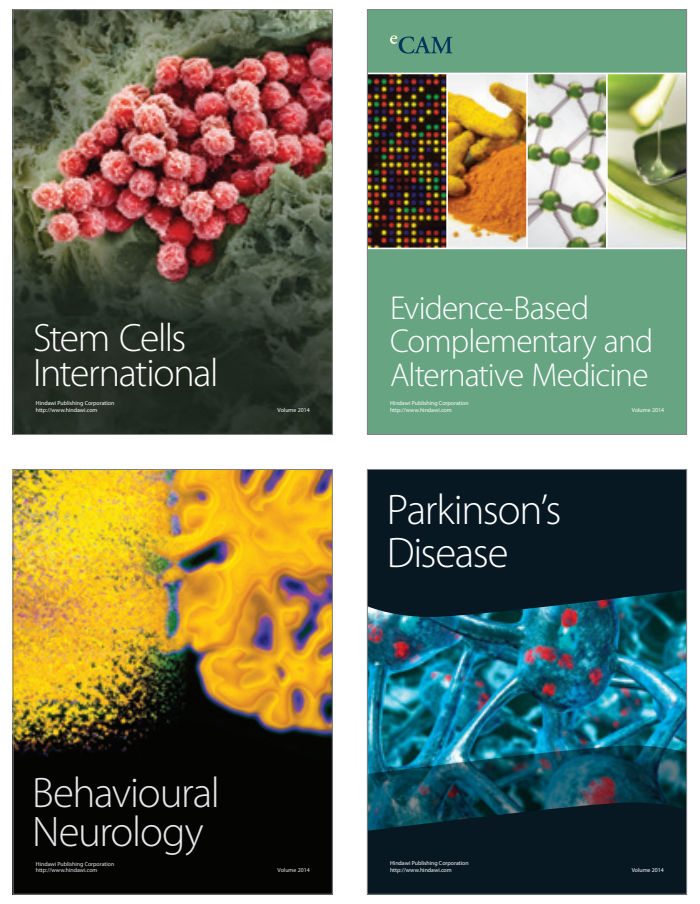
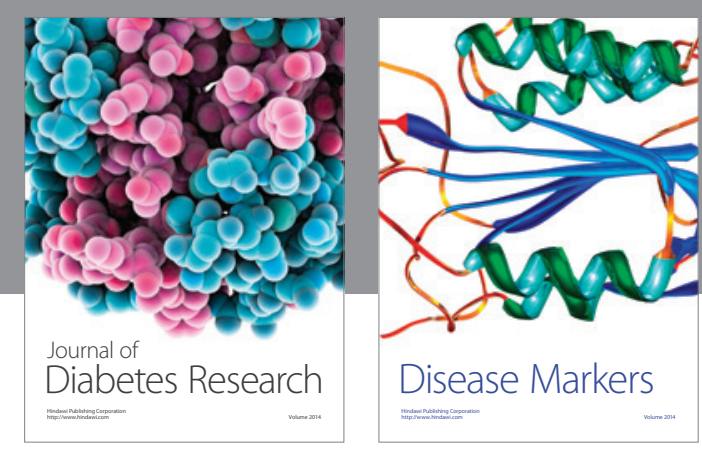

Disease Markers
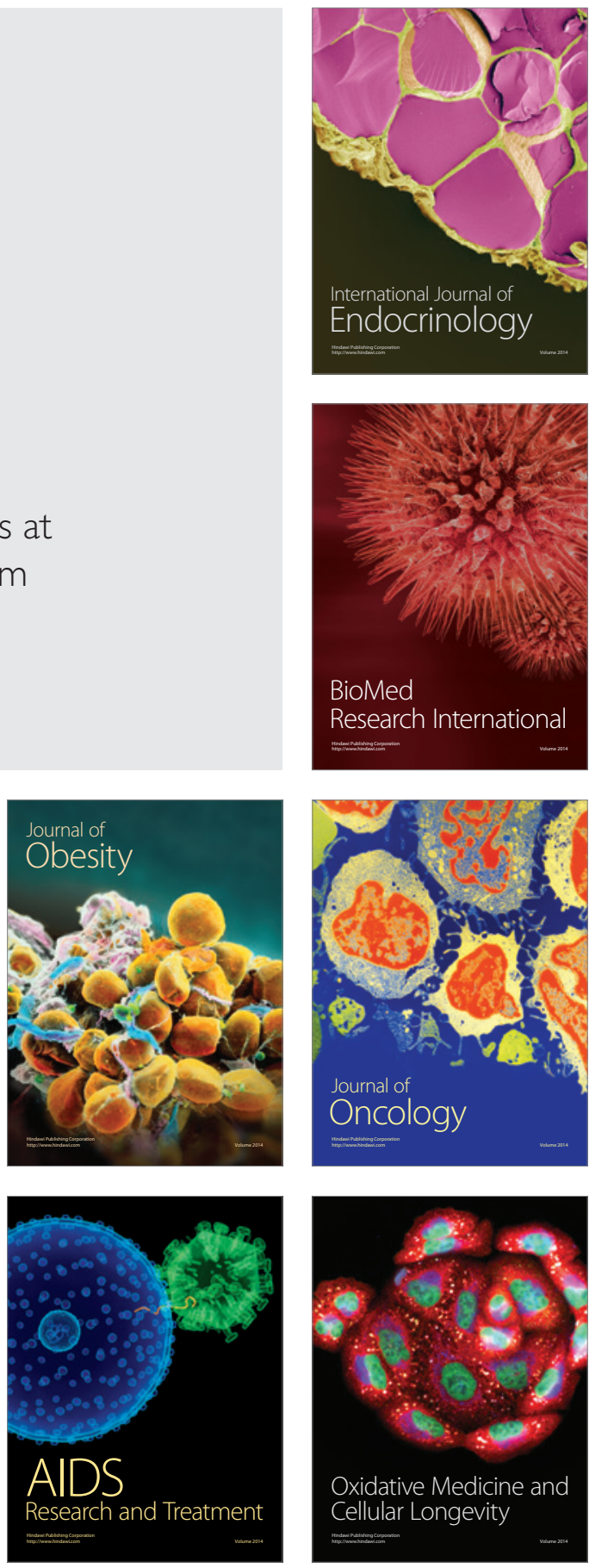